\title{
Treatment patterns and outcomes in the prophylaxis of chemotherapy-induced (febrile) neutropenia with biosimilar filgrastim (the MONITOR-GCSF study)
}

\author{
Pere Gascón $^{1}$ - Matti Aapro ${ }^{2}$ Heinz Ludwig ${ }^{3}$ - Carsten Bokemeyer ${ }^{4}$. \\ Mario Boccadoro $^{5}$ - Matthew Turner ${ }^{6}$ - Kris Denhaerynck ${ }^{7} \cdot$ Karen MacDonald $^{7}$. \\ Ivo Abraham ${ }^{7,8}$
}

Received: 4 March 2015 / Accepted: 17 July 2015 /Published online: 27 August 2015

(C) Springer-Verlag Berlin Heidelberg 2015

\begin{abstract}
Purpose The purpose of this study is to examine the realworld treatment patterns and outcomes of chemotherapyinduced (febrile) neutropenia (chemotherapy-induced (CIN)/ febrile neutropenia (FN)) prophylaxis with biosimilar filgrastim $\left(\right.$ Zarzio $\left.^{\circledR}\right)$.

Methods MONITOR-GCSF is an international (12 countries), multi-center (140), prospective (max. six cycles), observational, open-label, pharmaco-epidemiologic study of cancer patients $(n=1447)$ treated with myelosuppressive chemotherapy across a total of 6,213 cycles and receiving prophylaxis with Zarzio $^{\circledR}$. Data were analyzed using both the patient and cycle as unit of analysis.

Results Most (72.3\%) received primary prophylaxis; dosed mainly $(53.2 \%)$ at 30 MIU but differentiated by weight,
\end{abstract}

Prior dissemination: interim results of the MONITOR-GCSF study were presented at the 2012 (New York, NY, USA) and 2014 (Miami, FL, USA) MASCC congresses. Related abstracts:

Gascón P, Boccadoro M, Bokemeyer C et al. (2012) Support Care Cancer 20(Suppl 1):S206

Aapro M, Ludwig H, Bokemeyer C et al. (2012) Support Care Cancer 20(Suppl 1):S208

Aapro M, Ludwig H, Gascón P (2012) Support Care Cancer 2012;22(Suppl 1):S221

Ivo Abraham

abraham@pharmacy.arizona.edu

1 Division of Medical Oncology, Department of

Hematology-Oncology, Hospital Clínic de Barcelona, University of Barcelona, Barcelona, Spain

2 Institut Multidisciplinaire d'Oncologie, Clinique de Genolier, Genolier, Switzerland

3 Medizinische Abteilung I-Onkologie und Haematologie, Wilhelminenspital, Wien, Austria

chemotoxicity, and tumor type; and mainly (53.2\%) initiated in the $24-72 \mathrm{~h}$ post-chemotherapy window but differentiated by prophylaxis type, tumor type, and chemotoxicity and for modal/median duration of 5 days. Relative to European Organisation for Research and Treatment of Cancer (EORTC) guidelines, $56.6 \%$ were correctly prophylacted, $17.4 \%$ under-prophylacted, and $26.0 \%$ over-prophylacted. The following incidence rates were recorded: CIN grade $413.2 \%$ of patients and $3.9 \%$ of cycles, FN $5.9 \%$ of patients and $1.4 \%$ of cycles, CIN/FN-related hospitalizations $6.1 \%$ of patients and $1.5 \%$ of cycles, CIN/FN-related chemotherapy disturbances $9.5 \%$ of patients and $2.8 \%$ of cycles, and composite outcomes index $22.3 \%$ of patients and $6.7 \%$ of cycles. Rates varied by type of prophylaxis and tumor, chemotoxicity, initiation day, and prophylaxis duration. There were 1834

5 Dipartimento di Oncologia e Ematologia, Azienda Ospedaliero Universitaria S. Giovanni Battista di Torino, Torino, Italy

6 Sandoz Biopharmaceuticals, Holzkirchen, Germany

7 Matrix45, Tucson, AZ, USA

8 Center for Health Outcomes and PharmacoEconomic Research, University of Arizona, Drachman Hall B306H, 1295 N Martin, Tucson, AZ 85721, USA 
musculoskeletal events with $24.7 \%$ of patients reporting bone pain of any grade (mostly mild to moderate), and 148 adverse drug reactions, including 4 serious, were recorded in 76 patients.

Conclusions The clinical and safety outcomes are well within the range of historically reported data for originator filgrastim underscoring the clinical effectiveness and safety of biosimilar filgrastim in daily clinical practice.

Keywords Chemotherapy-induced neutropenia $\cdot$ Febrile neutropenia $\cdot$ Prophylaxis · Granulocyte colony-stimulating factor $\cdot$ Filgrastim $\cdot$ Biosimilar

\section{Introduction}

Chemotherapy-induced (CIN) and febrile neutropenia (FN) are potentially life-threatening complications of myelosuppressive chemotherapy, may often require hospitalization, and may result in disruptions to the planned chemotherapy regimen [1-5]. Known risk factors for CIN/FN enable clinicians to risk-stratify patients and initiate prophylaxis with granulocyte colony-stimulating factors (GCSF) [1, 3, 6-11].

GCSFs are biological growth factors that stimulate the production of white blood cells by promoting the proliferation, differentiation, and activation of neutrophils in the bone marrow [12]. The efficacy of standard and pegylated agents in FN prophylaxis is well established in terms of decreasing the risk of FN, the severity and duration of FN episodes, and chemotherapy disturbances - with no sustained evidence of superiority of either formulation $[9,10,13-17]$.

Following the patent expiration for filgrastim (Neupogen ${ }^{\circledR}$, Amgen) in Europe in 2006, several biosimilar agents have been approved by the European Medicines Agency, including EP-2006 (Zarzio ${ }^{\circledR}$, Filgrastim Hexal ${ }^{\circledR}$; Sandoz/Novartis; hereafter, Zarzio $\left.{ }^{\circledR}\right)$. A biosimilar or "similar biological medicinal product" is a "copy version of an already authorized biochemical medicinal product with demonstrated similarity in physicochemical characteristics, efficacy and safety, based on a comprehensive comparability exercise" [18 (p. 691)]. The clinical development of Zarzio ${ }^{\circledR}$ has been summarized [19] and reviewed in terms of clinical efficacy and safety $[20,21]$. Initial clinical experience suggests an effectiveness and safety profile similar to that of the originator product in core and extrapolated indications [22-25].

The MONITOR-GCSF study examined the real-world patterns, outcomes, and associated determinants of Zarzio ${ }^{\circledR}$ prophylaxis in cancer patients receiving myelosuppressive chemotherapy [26, 27]. Being European in scope, the study was framed within the European Organisation for Research and Treatment of Cancer (EORTC) guidelines for the use of GCSF in CIN/FN prophylaxis $[9,10]$, which specify that the myelotoxicity and associated FN risk of patients' regimens be assessed at each cycle. Primary prophylaxis is recommended for regimens with an FN risk $\geq 20 \%$ and for regimens with a risk of $10-20 \%$ if patients present with specific risk factors. GCSF prophylaxis at cycle 1 is not recommended if a regimen's FN risk is $<10 \%$. We report here on the treatment patterns and associated outcomes of CIN/FN prophylaxis with Zarzio $^{\circledR}$ in 1,447 patients from 140 cancer centers in $12 \mathrm{Eu}-$ ropean countries.

\section{Methods}

The methodology of MONITOR-GCSF has been described elsewhere [26, 27]. Methodology elements are summarized below.

\section{Design}

MONITOR-GCSF was an international, multi-center, prospective, observational, open-label, pharmaco-epidemiologic study of cancer patients treated with myelosuppressive chemotherapy regimens whose treating physicians prescribed $\mathrm{CIN} / \mathrm{FN}$ prophylaxis with Zarzio ${ }^{\circledR}$ per their best clinical judgment. Patients were recruited from 140 centers in 12 European countries: Austria (3 centers), Belgium (2), Czech Republic (5), France (34), Germany (27), Hungary (8), Italy (23), Poland (14), Romania (7), Spain (11), Switzerland (2), and the UK (4).

Eligible were male or female adults (age $\geq 18$ ) with stage 3 or 4 breast, ovarian, bladder, or lung cancer; metastatic prostate cancer; stage 3 or 4 diffuse large B cell lymphoma or multiple myeloma; and receiving primary or secondary prophylaxis with Zarzio ${ }^{\circledR}$. The safety sample consisted of patients who received at least one dose of Zarzio ${ }^{\circledR}$. The evaluable sample was limited to patients for whom at least initiation cycle data and follow-up/outcome data were available.

\section{Data model}

At enrollment: demographics, anthropometrics, medical and cancer history, prior cancer treatments, histology, history of (repeated) infections, and CIN/FN history.

At all visits: chemotherapy regimen, including changes; surgery, including changes; performance status; current and recent infections; CIN/FN episodes and associated hospitalizations; antibiotic prophylaxis; blood and urine cultures; Zarzio $^{\circledR}$ prophylaxis; selected concomitant medications; adherence; hematology parameters; clinical events; and adverse drug reactions (ADRs).

At study end: performance status, current and recent infections, CIN/FN episodes and associated hospitalizations, blood and urine cultures, hematology parameters, clinical events, and ADRs. 


\section{Other indices}

\section{Prophylaxis intensity}

The study followed the decision schematic in the 2010 EORTC guidelines for primary prophylaxis with GCSF, which were amended per ad hoc expert consensus to include trajectories for secondary prophylaxis. Patients' were graded accordingly as under-prophylacted, correctly prophylacted, or over-prophylacted.

\section{Patient risk score}

This score quantifies the eight risk factors that, according to the EORTC guidelines, may increase FN risk. Each factor was weighted by expert consensus: 3 to age $\geq 65$ years and prior FN history; 1.5 to advanced disease and poor performance and/or nutritional status; and 0.5 to no antibiotic prophylaxis, female gender, hemoglobin $<12 \mathrm{~g} / \mathrm{dL}$, and renal, cardiovascular, or liver disease. The sum of the weighted risk factors constituted the patient risk score (PRS) (range 0-11).

\section{Outcomes}

We report clinical outcomes using (a) patients and (b) chemotherapy cycles as the unit of analysis. Patient-level data enable analysis of the association of outcomes with prophylaxis type and chemotoxicity. Incidence refers to a given outcome "ever" experienced during the study. As patients enrolled at different cycles and were in the study for varying amounts of time, we also recorded outcomes at the cycle level. This permits analysis of the association with day of initiation and duration of prophylaxis. Cycle-level analysis evaluates the occurrence of an outcome in a given cycle or, in the case of chemotherapy disturbances, in a cycle subsequent to one in which a CIN/FN event was recorded (lag 1).

The outcomes of interest included the following: rate of CIN of any grade (CIN1/4), grades 3/4 combined (CIN3/4), grade 4 (CIN4), and FN; CIN/FN-related hospitalizations; CIN/FN-related chemotherapy disturbance one cycle after a CIN/FN event (lag 1); and a CIN/FN-related composite outcome that included any occurrence of CIN4, FN, CIN/FNrelated hospitalizations, and/or CIN/FN-related chemotherapy disturbances.

\section{Specialized statistical issues}

The statistical dependence inherent to the structure of the cycle data being "nested" under patients was taken into account using generalized estimating equations. This procedure adjusts standard errors based on the observed withincluster correlation.

We applied time-to-event modeling methods to estimate the probability of patients developing CIN4 or FN, being hospitalized, or experiencing chemotherapy disturbances due to $\mathrm{CIN} / \mathrm{FN}$ in later cycles. For patients receiving primary prophylaxis, we estimated the probability of any one of these outcomes occurring in the cycle in which Zarzio ${ }^{\circledR}$ was initiated and the remaining five chemotherapy cycles. For patients receiving secondary prophylaxis in cycle 2 , we estimated the probability of any of these outcomes occurring in cycles 2 through 6 . The secondary prophylaxis exercise was limited to patients prophylacted in cycle 2 because too few patients were initiated in cycle 3 or later. In both exercises, chemotherapy disturbances were not estimated for the initiation cycle as such disturbances always occur with a cycle lag $=1$.

\section{Human rights}

This study was approved by the ethical review committees of participating centers in accordance with national laws and regulations. Patients provided written informed consent.

\section{Results}

\section{Patients}

In total, 1,496 patients were enrolled yielding an evaluable sample of 1,447 patients (Fig. 1a). The majority of patients $(72.3 \%)$ were enrolled in cycle 1 (Fig. 1b). The sample was predominantly female $(61.2 \%)$ (Table 1$)$. Median age was 62 years. Most patients $(89.1 \%)$ had an ECOG $\leq 1$ score. Safety-relevant history included musculoskeletal pain (15.5\%), headaches (1.9\%), and bleeding (1.2\%).

Most patients $(77.2 \%)$ had a solid tumor, mainly breast (32.2\%) or lung $(23.8 \%)$ cancer. The most prevalent hematological malignancy was lymphoma (16.9\%). Proportions of stage 3 versus 4 disease varied across tumor types, with a majority of oncological patients $(59.1 \%)$ having stage 4 but equal distribution of either stage in hematological patients. One third of patients $(35.8 \%)$ were cancer-treatment-naive; one third $(31.8 \%)$ had received at least one line of chemotherapy, while one third (32.6\%) had undergone surgery.

Of the 460 patients with prior chemotherapy, 106 (23.0\%) had experienced $\geq 1$ CIN4 episodes, including 27 (5.9\%) with episodes classified FN. Only $55(12.0 \%)$ had received GCSF treatment. Thirty-three (7.2\%) required hospitalization, and 48 (10.4\%) experienced chemotherapy disturbances.

In the 10-20\% subsample, there were proportionately more patients with the risk factor of age $\geq 65$ ( $p=0.043)$, advanced disease $(p<0.001)$, and no antibiotic prophylaxis $(p=$ $0.019)$ than in the rest of the sample. The PRS mean $( \pm$ SD) was $2.85 \pm 1.96$, with patients receiving secondary prophylaxis having a higher PRS ( $p=0.007)$. About equal proportions of patients were being treated with regimens with $\mathrm{FN}$ risk $>20 \%$ $(44.3 \%)$ or $10-20 \%(45.0 \%)$. 
Fig. 1 a Subject enrollment and b follow-up a

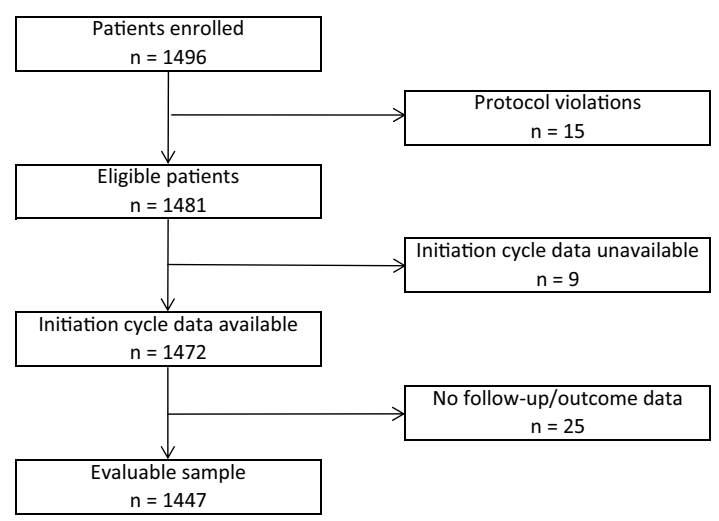

b

Follow-Up Visit Rates

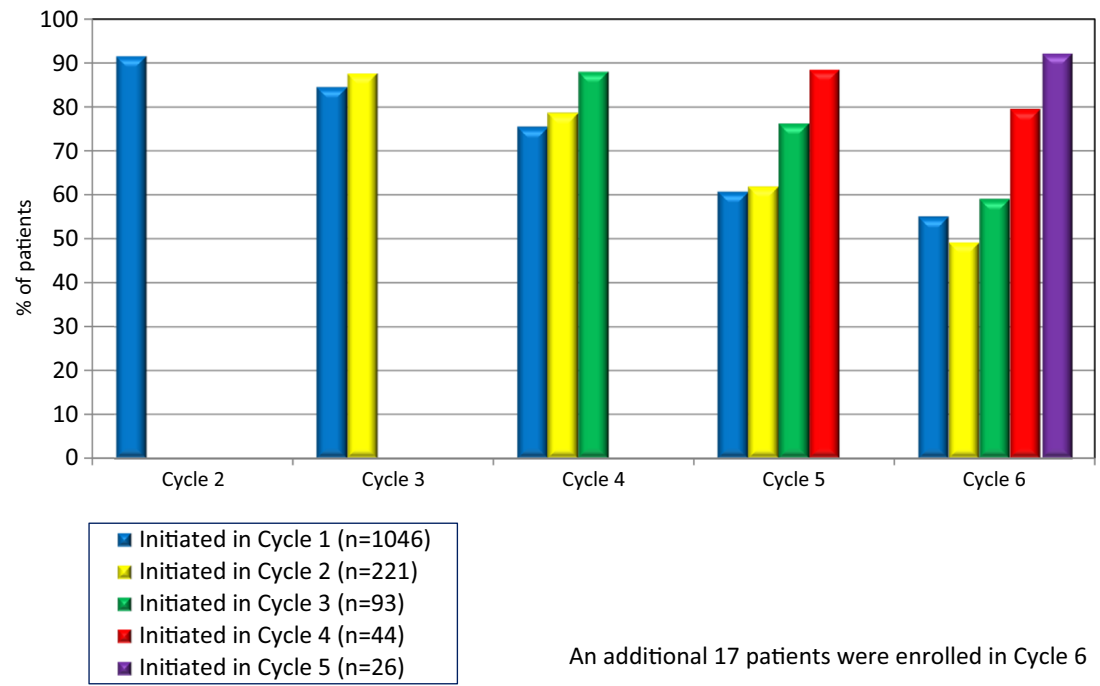

\section{Prophylaxis}

Zarzio ${ }^{\circledR}$ was initiated as primary prophylaxis in $72.3 \%$ and as secondary prophylaxis in $27.7 \%$ of patients (Table 2). Relative to the EORTC guidelines, $56.6 \%$ of patients were correctly prophylacted, $17.4 \%$ under-prophylacted, and $26.0 \%$ over-prophylacted (Fig. 2). Under-prophylaxis occurred when secondary prophylaxis was given, but primary prophylaxis was recommended by the guidelines, whereas overprophylaxis occurred when primary prophylaxis was given when not recommended by the guidelines.

Proportionately more patients were administered a dose of 30 MIU $(p<0.001)$. This was not a function of type of prophylaxis $(p=$ n.s. $)$ but of body weight $(p<0.001)$, chemotoxicity $(p=0.007)$, and tumor type $(p<0.001)$.

In half of patients $(53.2 \%)$, Zarzio ${ }^{\circledR}$ was initiated in days $1-3$ following chemotherapy $(\mathrm{M} \pm \mathrm{SD}=3.08 \pm 2.98)$, yet $13.4 \%$ were exposed on the day of, and the remaining $33.4 \% \geq 4$ days following, chemotherapy. Half $(52.9 \%)$ of the hematological patients were initiated on days 4-8. Day of GCSF initiation tended to be earlier for patients with solid tumors $(p<0.001)$, receiving secondary prophylaxis $(p=$ 0.010 ), or on chemotherapy regimens with low or moderate FN risk $(p<0.001)$.

The modal duration of prophylaxis was 5 days ( $45.7 \%$ of patients); $72.7 \%$ received Zarzio ${ }^{\circledR}$ for $4-8$ days. Duration was longer with higher chemotoxicity $(p<0.001)$ but was independent of prophylaxis or tumor type (both $p=$ n.s.).

\section{Clinical outcomes}

Patient level

In total, 504 (34.8\%) patients experienced one or more (ever) CIN1/4 episodes (Table 3). Rates were higher among patients receiving secondary prophylaxis $(p<0.001)$ but were independent of chemotherapy regimen. The CIN $3 / 4$ and CIN 4 rates were 22.9 and $13.2 \%$, respectively, and were independent of prophylaxis type (both $p=$ n.s.) but rose with chemotoxicity (both $p<0.03$ ). The FN incidence was $5.9 \%$, was independent of prophylaxis type but was associated with chemotoxicity $(p<0.001)$. 
Table 1 Demographics, clinical status, and cancer and CIN/FN history at enrollment

Demographics

Gender $(n, \%)$
Ethnicity $(n, \%)$
Age $(M \pm \mathrm{SD}$, median $)$
Country

Biometrics

Height $(\mathrm{cm})(M \pm \mathrm{SD}$, median)

Weight $(\mathrm{kg})(M \pm \mathrm{SD}$, median)

BMI $(M \pm \mathrm{SD}$, median)

ECOG performance status $(n, \%)$

Comorbidities $(n, \%)$

Hypertension

Coronary disease

Cardiac arrhythmia

Peripheral vascular disease

Cardiac failure

Other cardiovascular

Anemia

Type 2 diabetes

Type 1 diabetes

COPD

Other respiratory

Allergies

Hepatitis

Gastro-intestinal disease

Renal disease

Neurological disease

Cerebrovascular disease

Relevant medical history $(n, \%)$

Bone pain

Joint pain

Muscle pain

Headache

Epistaxis

$\begin{array}{lll}\text { Male } & 561 & 38.8 \% \\ \text { Female } & 886 & 61.2 \% \\ \text { Caucasian } & 1042 & 99.0 \% \\ \text { Other } & 10 & 1.0 \% \\ \text { Not reported }^{\mathrm{a}} & 395 & \\ & 61.3 \pm 11.8 & 62 \\ \text { Austria } & 27 & 1.9 \% \\ \text { Belgium } & 3 & 0.2 \% \\ \text { Czech Republic } & 50 & 3.4 \% \\ \text { France } & 395 & 27.3 \% \\ \text { Germany } & 145 & 10.0 \% \\ \text { Hungary } & 143 & 9.9 \% \\ \text { Italy } & 175 & 12.1 \% \\ \text { Poland } & 286 & 19.8 \% \\ \text { Romania } & 64 & 4.4 \% \\ \text { Spain } & 116 & 8.0 \% \\ \text { Switzerland } & 11 & 0.8 \% \\ \text { UK } & 32 & 2.2 \%\end{array}$

$\begin{array}{ll}165.6 \pm 8.5 & 165 \\ 72.1 \pm 15.0 & 70 \\ 26.3 \pm 5.0 & 25.7 \\ 553 & 40.9 \% \\ 652 & 48.2 \% \\ 121 & 8.9 \% \\ 26 & 1.9 \% \\ 1 & 0.1 \%\end{array}$

$\begin{array}{ll}116 & 8.0 \% \\ 78 & 5.4 \% \\ 31 & 2.1 \% \\ 28 & 1.9 \% \\ 8 & 0.6 \%\end{array}$


Table 1 (continued)

Gastro-intestinal bleeding

Skin hemorrhage

Tumor type $(n, \%)^{\mathrm{b}} \quad$ All patients

Solid

Breast

Lung

Ovarian

Prostate

Bladder

Hematological

Lymphoma

Multiple myeloma

Prior cancer treatments $(n, \%)^{\mathrm{c}}$

None

Surgery

Chemotherapy

Of these:

Adjuvant
In metastatic setting
Of these: prior lines of chemo ${ }^{b}$

$$
1
$$

2 $\geq 3$

Radiation therapy

Hormonal therapy

Targeted therapy

Bone marrow transplant

Other (incl. CAM)

History of repeated infections $(n, \%)$

\section{Of these:}

Chronic

Recurrent acute
Of these: number of episodes in past 3 months

1

$\geq 3$

$\mathrm{CIN} / \mathrm{FN}$ in prior line of chemo $(n, \%)$

Prior CIN grade four episode(s)

Of these: number of episodes ${ }^{b}$

1

2

$\geq 3$

Prior FN episode(s)

CIN/FN-related hospitalization

$\mathrm{CIN} / \mathrm{FN}$-related chemotherapy disturbances ${ }^{\mathrm{c}}$

Any disturbance

Chemotherapy delay

Chemotherapy dose reduction

Chemotherapy cancellation

Prior GCSF therapy
7

1117

466

345

140

102

64

330

245

85

518

472

460

196

206

103

54

42

274

198

45

12

25

35

8

27

15

7

3

106

79

5

15

27

33

48

48

41

12

5

55
1

$0.5 \%$

$0.1 \%$

Stage 3

$77.2 \%$

454

267

101

63

7

16

156

103

53

\begin{tabular}{llll}
\multicolumn{5}{c}{ Stage 4} \\
$40.9 \%$ & 655 & $59.1 \%$ \\
$57.9 \%$ & 194 & $42.1 \%$ \\
$29.5 \%$ & 241 & $70.5 \%$ \\
$45.0 \%$ & 77 & $55.0 \%$ \\
$6.9 \%$ & 95 & $93.1 \%$ \\
$25.0 \%$ & 48 & $75.0 \%$ \\
$50.8 \%$ & 151 & $49.2 \%$ \\
$43.3 \%$ & 135 & $56.7 \%$ \\
$76.8 \%$ & 16 & $23.2 \%$
\end{tabular}

$35.8 \%$

$32.6 \%$

$31.8 \%$

$42.6 \%$

$44.8 \%$

$51.8 \%$

$27.1 \%$

$21.1 \%$

$18.9 \%$

$13.7 \%$

$3.1 \%$

$0.8 \%$

$1.7 \%$

$2.4 \%$

$22.9 \%$

$77.1 \%$

$46.9 \%$

$21.9 \%$

$9.5 \%$

$\%$ Prior chemo

$23.0 \%$

$\%$ Episodes

$79.8 \%$

$5.0 \%$

$15.2 \%$

$\%$ Prior chemo \% Prior CIN4

$5.9 \% \quad 25.5 \%$

$7.2 \% \quad 31.1 \%$

$10.4 \% \quad 45.3 \%$

$8.9 \% \quad 38.7 \%$

$2.6 \% \quad 11.3 \%$

$1.1 \% \quad 4.7 \%$

$12.0 \% \quad 51.9 \%$ 
Table 1 (continued)

\begin{tabular}{|c|c|c|c|c|c|c|}
\hline \multicolumn{7}{|c|}{ Chemotherapy during study $(n, \%)$} \\
\hline & Chemotherapy cycle at entry into study & & & & & \\
\hline & 1 & 1046 & $72.3 \%$ & & & \\
\hline & 2 & 221 & $15.3 \%$ & & & \\
\hline & 3 & 93 & $6.4 \%$ & & & \\
\hline & 4 & 44 & $3.0 \%$ & & & \\
\hline & 5 & 26 & $1.8 \%$ & & & \\
\hline & 6 & 17 & $1.2 \%$ & & & \\
\hline & Myelotoxicity-associated FN risk & & & & & \\
\hline & $<10 \%$ & 154 & $10.7 \%$ & & & \\
\hline & $10-20 \%$ & 650 & $45.0 \%$ & & & \\
\hline & $>20 \%$ & 640 & $44.3 \%$ & & & \\
\hline FN risk factors $(n, \%)$ & & All patients & & $10-2$ & & $p$ \\
\hline & High risk & & & & & \\
\hline & Age $\geq 65$ years & 598 & $41.3 \%$ & 290 & $44.6 \%$ & 0.043 \\
\hline & Increased risk & & & & & \\
\hline & Advanced disease $^{\mathrm{d}}$ & 187 & $13.7 \%$ & 127 & $20.8 \%$ & $<0.001$ \\
\hline & History of FN & 27 & $1.9 \%$ & 13 & $2.0 \%$ & n.s. \\
\hline & No antibiotic prophylaxis & 1261 & $87.8 \%$ & 590 & $91.3 \%$ & 0.019 \\
\hline & Other factors & & & & & \\
\hline & Poor performance and/or nutritional status & 178 & $13.1 \%$ & 82 & $13.3 \%$ & n.s. \\
\hline & Female gender & 886 & $61.2 \%$ & 376 & $57.8 \%$ & n.s. \\
\hline & Hemoglobin $<12 \mathrm{~g} / \mathrm{dL}$ & 482 & $39.6 \%$ & 243 & $45.9 \%$ & n.s. \\
\hline & Renal, cardiovascular, or liver disease & 304 & $23.1 \%$ & 156 & $26.9 \%$ & n.s. \\
\hline Patient risk score (PRS) & & $M \pm \mathrm{SD}$ & Median & & & $p$ \\
\hline & All patients & $2.85 \pm 1.96$ & 2.5 & & & \\
\hline & By type of prophylaxis & & & & & 0.007 \\
\hline & Primary & $2.73 \pm 1.92$ & 2.5 & & & \\
\hline & Secondary & $3.14 \pm 2.01$ & 3.0 & & & \\
\hline Concurrent cancer treatm & nents during study $(n, \%)^{\mathrm{c}}$ & & & & & \\
\hline & Surgery & 100 & $6.9 \%$ & & & \\
\hline & Radiation therapy & 102 & $7.1 \%$ & & & \\
\hline & Hormonal therapy & 85 & $5.9 \%$ & & & \\
\hline & Targeted treatment & 89 & $6.2 \%$ & & & \\
\hline & Bone marrow transplant & 31 & $2.1 \%$ & & & \\
\hline & Other (incl. CAM) & 98 & $6.8 \%$ & & & \\
\hline
\end{tabular}

$B M I$ body mass index, $C A M$ complementary and alternative medicine, $C I N$ chemotherapy-induced neutropenia, $C O P D$ chronic obstructive pulmonary disease, ECOG Eastern Cooperative Oncology Group, $F N$ febrile neutropenia, GCSF granulocyte colony-stimulating factor

${ }^{a}$ French regulations do not permit recording of ethnicity in clinical studies

${ }^{\mathrm{b}}$ Frequencies as reported

${ }^{\mathrm{c}}$ Not mutually exclusive. Patient may have had more than one

${ }^{\mathrm{d}}$ Stage 3 (stage 3 or 4 if multiple myeloma) and prior chemo in metastatic setting

Eighty-eight patients $(6.1 \%)$ were hospitalized. Hospitalization was independent of prophylaxis type and chemotoxicity (both $p=$ n.s.). The chemotherapy regimen of 138 patients $(9.5 \%)$ was disturbed, and this more so among secondary prophylaxis patients (both $p<0.001$ ). Chemotherapy disturbances were independent of chemotoxicity.

Three hundred twenty-three patients $(22.3 \%)$ scored positive on the CIN/FN-related composite outcome. This was independent of prophylaxis type $(p=$ n.s.), but associated with chemotoxicity $(p=0.043)$.

Table 4 summarizes, for patients who received primary prophylaxis, the number who experienced each event; the associated cumulative probabilities derived from time-to-event analyses as patients progressed through chemotherapy cycles; and, as an index of variability, the difference in probabilities between the highest and lowest estimates in a series. Similar data are presented for patients on secondary prophylaxis, but analysis was limited to patients initiated on Zarzio ${ }^{\circledR}$ in cycle 2, as statistically too few patients received secondary prophylaxis at later time points. The frequencies indicate the number of patients who experienced one of the four outcomes while the associated probabilities were derived from the time-to-event modeling calculations.

Among patients receiving primary prophylaxis, the most infrequently expected event over up to six cycles of chemotherapy was hospitalization in cycle 1 with a modeled occurrence of $2 \%$ of patients. The most frequently observed 
Table 2 Zarzio ${ }^{\circledR}$ prophylaxis patterns

Type of prophylaxis

Primary (\%)

$72.3 \%$

Dose

All patients

$30 \mathrm{MIU} /$ day

$48 \mathrm{MIU} /$ day

Other

By type of prophylaxis

$30 \mathrm{MIU} /$ day

$48 \mathrm{MIU} /$ day

By patient weight

$30 \mathrm{MIU} /$ day

$48 \mathrm{MIU} /$ day

By chemotoxicity

$30 \mathrm{MIU} /$ day

$48 \mathrm{MIU} /$ day

By tumor type

$30 \mathrm{MIU} /$ day

$48 \mathrm{MIU} /$ day

Day of initiation ${ }^{\mathrm{a}}$

$\begin{array}{ll}0 & 13.4 \% \\ 1 & 30.7 \% \\ 2 & 13.4 \% \\ 3 & 9.1 \% \\ 4 & 4.6 \% \\ 5 & 6.8 \% \\ 6 & 6.7 \% \\ 7 & 7.2 \% \\ 8 & 3.9 \% \\ 9 & 1.0 \% \\ 10 & 0.8 \% \\ 11 & 0.6 \% \\ \geq 12 & 1.8 \%\end{array}$

All patients

By type of prophylaxis

Primary

Secondary

By chemotoxicity

$<10 \%$

$10-20 \%$

$\geq 20 \%$

By tumor type

Oncology

Hematology

$27.7 \%$
Secondary $(\%)$

$P$ value

$<0.001$

$<0.001$

$53.2 \%$

$46.0 \%$

$0.8 \%$

Primary

Secondary

n.s.

$53.7 \% \quad 53.2 \%$

$46.3 \% \quad 46.8 \%$

$\leq 65 \mathrm{~kg} \quad>65 \mathrm{~kg}$

$66.1 \% \quad 45.9 \%$

$33.9 \% \quad 54.1 \%$

$<10 \%$

$65.4 \%$

$34.6 \%$

Oncology

$49.8 \%$

$50.2 \%$

10-20\%

$>20 \%$

0.007

$54.3 \%$

$50.7 \%$

$45.7 \%$

$49.3 \%$

Hematology

$<0.001$

$66.7 \%$

$33.3 \%$

Oncology

Hematology

$<0.001$

$16.0 \%$

$4.2 \%$

$33.6 \% \quad 20.4 \%$

$14.9 \% \quad 8.0 \%$

$9.8 \% \quad 6.9 \%$

$4.1 \% \quad 6.2 \%$

$6.7 \% \quad 7.2 \%$

$3.4 \% \quad 18.6 \%$

$5.8 \% \quad 12.2 \%$

$2.5 \% \quad 8.7 \%$

$1.0 \% \quad 0.9 \%$

$0.6 \% \quad 1.5 \%$

$0.5 \% \quad 1.1 \%$

$1.1 \% \quad 4.1 \%$

Mean SD

3.08

2.98

Median

$p$

2

3.19

3.00

0.010

2.63

2.87

2

1

2.31

2.95

2.85

3.00

2.96
2.60

4.76
2.70

3.30
$<0.001$

$<0.001$

1

2

2

2

5 
Table 2 (continued)

Duration of prophylaxis (days)

$\begin{array}{ll}1 & 3.6 \% \\ 2 & 5.7 \% \\ 3 & 12.3 \% \\ 4 & 7.1 \% \\ 5 & 45.7 \% \\ 6 & 6.5 \% \\ 7 & 11.5 \% \\ 8 & 1.9 \% \\ 9 & 0.9 \% \\ 10 & 2.0 \% \\ 11 & 0.2 \% \\ 12 & 0.3 \% \\ 13 & 0.1 \% \\ 14 & 1.8 \% \\ \geq 15 & 0.4 \%\end{array}$

\begin{tabular}{|c|c|c|c|c|}
\hline & Mlean & & Median & $p$ value \\
\hline All patients & 5.11 & 2.32 & 5 & \\
\hline By type of prophylaxis & & & & n.s. \\
\hline Primary & 5.10 & 2.21 & 5 & \\
\hline Secondary & 5.17 & 2.68 & 5 & \\
\hline By chemotoxicity & & & & $<0.001$ \\
\hline$<10 \%$ & 4.59 & 2.41 & 5 & \\
\hline $10-20 \%$ & 4.98 & 2.23 & 5 & \\
\hline$\geq 20 \%$ & 5.33 & 2.35 & 5 & \\
\hline By tumor type & & & & n.s. \\
\hline Oncology & 5.14 & 2.24 & 5 & \\
\hline Hematology & 5.03 & 2.56 & 5 & \\
\hline
\end{tabular}

${ }^{\mathrm{a}}$ Zarzio $^{\circledR}$ initiation expressed in days after chemotherapy $(0=$ same day; $1=1$ day after, $2=2$ days after, etc. $)$

Fig. 2 Treatment decision relative to EORTC guidelines

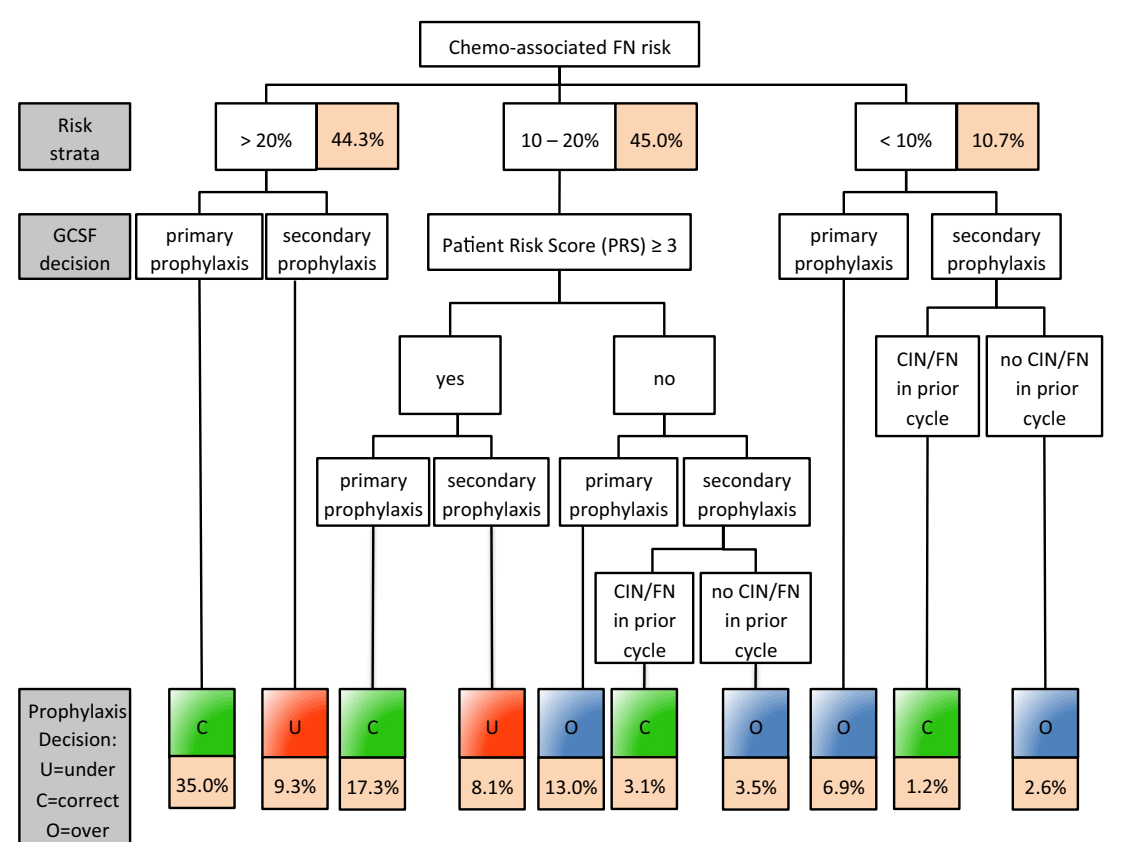

Shaded boxes indicate percentages in each category in the MONITOR-GCSF sample. 
Table 3 Clinical outcomes at the patient and cycle levels

\begin{tabular}{|c|c|c|c|c|c|c|c|c|c|c|}
\hline \multicolumn{11}{|l|}{ Unit of analysis: patient } \\
\hline & \multicolumn{4}{|c|}{ By prophylaxis } & & & \multicolumn{4}{|c|}{ By chemotoxicity } \\
\hline & \multicolumn{2}{|c|}{ All patients $(n, \%)$} & Primary (\%) & Secondary $(\%)$ & \multicolumn{2}{|l|}{$p$} & $<10 \%(\%)$ & $10-20 \%(\%)$ & $>20 \%(\%)$ & $p$ value \\
\hline \multicolumn{11}{|l|}{ Neutropenia episodes } \\
\hline CIN grades 1 through 4 & 504 & $34.8 \%$ & 32.1 & 41.9 & \multicolumn{2}{|l|}{0.001} & 29.9 & 36.0 & 34.8 & n.s. \\
\hline CIN grades 3 or 4 & 332 & $22.9 \%$ & 22.5 & 24.2 & \multicolumn{2}{|l|}{ n.s. } & 14.9 & 22.9 & 25.0 & 0.029 \\
\hline $\mathrm{CIN}$ grade 4 & 191 & $13.2 \%$ & 13.8 & 11.7 & \multicolumn{2}{|l|}{ n.s. } & 7.8 & 11.9 & 15.9 & 0.010 \\
\hline FN & 86 & $5.9 \%$ & 6.7 & 4.0 & \multicolumn{2}{|l|}{ n.s. } & 3.9 & 3.5 & 8.9 & $<0.001$ \\
\hline CIN/FN-related hospitalizations & 88 & $6.1 \%$ & 6.1 & 6.0 & \multicolumn{2}{|l|}{ n.s. } & 3.9 & 5.4 & 7.3 & n.s. \\
\hline \multicolumn{11}{|l|}{ CIN/FN-related chemotherapy disturbances ${ }^{\mathrm{a}}$} \\
\hline Any chemotherapy disturbance & 138 & $9.5 \%$ & 7.5 & 15.0 & \multicolumn{2}{|l|}{$<0.001$} & 8.4 & 10.8 & 8.6 & n.s. \\
\hline Chemotherapy dose reduction & 45 & $3.1 \%$ & 2.9 & 3.7 & \multicolumn{2}{|l|}{ n.s. } & 3.9 & 3.2 & 2.8 & n.s. \\
\hline Chemotherapy delay & 113 & $7.8 \%$ & 5.8 & 13.0 & \multicolumn{2}{|l|}{$<0.001$} & 7.1 & 8.9 & 6.9 & n.s. \\
\hline Chemotherapy cancellation & 3 & $0.2 \%$ & 0.1 & 0.5 & \multicolumn{2}{|l|}{ n.s. } & 0.0 & 0.0 & 0.5 & n.s. \\
\hline CIN/FN-related composite outcome ${ }^{\mathrm{b}}$ & 323 & $22.3 \%$ & 21.5 & 24.4 & \multicolumn{2}{|l|}{ n.s. } & 16.9 & 20.9 & 25.2 & 0.043 \\
\hline \multicolumn{11}{|l|}{ Unit of analysis: cycle } \\
\hline & & & \multicolumn{5}{|c|}{ By day of initiation of prophylaxis } & \multicolumn{3}{|c|}{ By duration of prophylaxis } \\
\hline & \multicolumn{2}{|c|}{ All cycles $(n, \%)$} & Day of chemo (\%) & $24-72$ h (\%) & $>72 \mathrm{~h}(\%) \mathrm{s}$ & $p$ & $1-3$ days $(\%)$ & $4-5$ days $(\%)$ & $\geq 6$ days & $p$ value \\
\hline \multicolumn{11}{|l|}{ Neutropenia episodes } \\
\hline CIN grades 1 through 4 & 1083 & $14.3 \%$ & 13.0 & 13.4 & 20.0 & $<0.001$ & 15.7 & 13.4 & 20.0 & $<0.001$ \\
\hline $\mathrm{CIN}$ grades 3 or 4 & 602 & $8.0 \%$ & 6.7 & 6.3 & 13.4 & $<0.001$ & 9.1 & 7.6 & 10.3 & 0.008 \\
\hline CIN grade 4 & 294 & $3.9 \%$ & 3.1 & 2.8 & 7.3 & $<0.001$ & 3.6 & 3.9 & 5.5 & 0.015 \\
\hline FN & 105 & $1.4 \%$ & 1.0 & 1.2 & 2.1 & 0.029 & 1.2 & 1.2 & 2.2 & 0.015 \\
\hline CIN/FN-related hospitalizations & 111 & $1.5 \%$ & 1.9 & 1.1 & 1.8 & n.s. & 1.2 & 1.4 & 1.8 & n.s. \\
\hline \multicolumn{11}{|l|}{$\mathrm{CIN} / \mathrm{FN}$-related chemotherapy disturbances ${ }^{\mathrm{a}}$} \\
\hline Any chemotherapy disturbance & 174 & $2.8 \%$ & 3.5 & 2.9 & 1.9 & n.s. & 2.0 & 2.1 & 4.7 & $<0.001$ \\
\hline Chemotherapy dose reduction & 46 & $0.7 \%$ & 1.1 & 0.6 & 0.9 & n.s. & 0.6 & 0.8 & 1.0 & n.s. \\
\hline Chemotherapy delay & 142 & $2.3 \%$ & 3.8 & 2.4 & 1.2 & $<0.001$ & 2.0 & 1.5 & 3.9 & $<0.001$ \\
\hline Chemotherapy cancellation & 3 & $0.1 \%$ & 0.0 & $<0.1$ & 0.1 & n.s. & 0.0 & 0.1 & 0.0 & n.s. \\
\hline CIN/FN-related composite outcome $\mathrm{b}^{\mathrm{b}}$ & 507 & $6.7 \%$ & 5.8 & 5.4 & 9.1 & $<0.001$ & 6.0 & 5.8 & 9.3 & $<0.001$ \\
\hline
\end{tabular}

${ }^{a}$ Type of chemotherapy disturbances is not a mutually exclusive variable. Any patient may have experienced more than one type. Measured with $10 N E-$ cycle lag

${ }^{\mathrm{b}}$ Includes any occurrence of CIN grade 4, FN, CIN/FN-related hospitalization, and/or CIN/FIN-related chemotherapy disturbance

outcome was a CIN4 episode at $16 \%$ over six cycles. Among patients on secondary prophylaxis, the most infrequently expected event over up to six cycles of chemotherapy was an FN episode in the initiation cycle (cycle 2 ) at $4 \%$ of patients, while the most frequent event modeled, just as in primary prophylaxis, was CIN4 over six cycles at $16 \%$.

\section{Cycle level}

CIN1/4, CIN3/4, CIN4, and FN episodes were recorded in, respectively, $14.3,8.0,3.9$, and $1.4 \%$ of cycles (Table 3). These rates were higher for prophylaxis initiated $>72 \mathrm{~h}$ following chemotherapy (all $p<0.03$ ) and for prophylaxis of $\geq 6$ day duration (all $p<0.02$ ).

CIN/FN-related hospitalizations occurred in $1.5 \%$ of cycles but was independent of initiation or duration of prophylaxis (both $p=$ n.s.). In total, $174(2.8 \%)$ chemotherapy cycles were disturbed, which was independent of day of initiation $(p=$ n.s. $)$ but higher for prophylaxis lasting $\geq 6$ days $(p<0.001)$. Chemotherapy delay was the most frequent disturbance $(2.3 \%)$ and was associated with prophylaxis initiation $(p<0.001)$ and duration $(p=0.001)$.

In total, 507 cycles $(6.7 \%)$ were positive on the CIN/FNrelated composite outcome. The rate was higher for cycles in which prophylaxis was initiated $>72 \mathrm{~h}(p<0.001)$ or lasted $\geq 6$ days $(p<0.001)$.

\section{Safety}

In the safety sample (1,496 patients with 6,392 cycles), 4271 events were observed in 777 (53.7 \%) patients (Table 5). Bone pain was the most prevalent $(24.7 \%)$ and was mostly mild to moderate. Five hundred twenty patients $(35.9 \%)$ experienced more than one event. Sixtyone patients died, mainly due to cancer $(67.2 \%)$. There were 148 ADRs reported $(2.3 \%$ of cycles) in $76(5.1 \%)$ patients. Most ADRs were mild or moderate $(83.8 \%$ ) and 
Table 4 Observed outcomes over the course chemotherapy cycles for patients receiving primary (Zarzio ${ }^{\circledR}$ initiated in cycle 1$)$ and secondary $\left(\right.$ Zarzio ${ }^{\circledR}$ initiated in cycle 2 only) prophylaxis and associated probabilities

\begin{tabular}{|c|c|c|c|c|c|c|c|c|c|c|c|c|c|}
\hline \multirow[t]{2}{*}{ Primary prophylaxis } & \multicolumn{2}{|c|}{ Cycle 1} & \multicolumn{2}{|c|}{ Cycle 2} & \multicolumn{2}{|c|}{ Cycle 3} & \multicolumn{2}{|c|}{ Cycle 4} & \multicolumn{2}{|c|}{ Cycle 5} & \multicolumn{2}{|c|}{ Cycle 6} & \multirow[t]{2}{*}{$\Delta P(\text { event })^{\mathrm{c}}$} \\
\hline & $n^{\mathrm{a}}$ & $P{\text { (event })^{\mathrm{b}}}$ & $n^{\mathrm{a}}$ & $P(\text { event })^{\mathrm{b}}$ & $n^{\mathrm{a}}$ & $P(\text { event })^{\mathrm{b}}$ & $n^{\mathrm{a}}$ & $P(\text { event })^{\mathrm{b}}$ & $n^{\mathrm{a}}$ & $P(\text { event })^{\mathrm{b}}$ & $n^{\mathrm{a}}$ & $P(\text { event })^{\mathrm{b}}$ & \\
\hline \multicolumn{14}{|l|}{ Patients with } \\
\hline CIN4 & 74 & 0.07 & 31 & 0.10 & 12 & 0.12 & 11 & 0.13 & 7 & 0.14 & 9 & 0.16 & 0.09 \\
\hline $\mathrm{FN}$ & 31 & 0.03 & 14 & 0.04 & 11 & 0.06 & 5 & 0.06 & 3 & 0.07 & 6 & 0.08 & 0.05 \\
\hline Hospitalization & 22 & 0.02 & 10 & 0.03 & 9 & 0.04 & 4 & 0.05 & 5 & 0.05 & 3 & 0.06 & 0.04 \\
\hline Chemotherapy disturbance & & & 36 & 0.04 & 17 & 0.06 & 10 & 0.07 & 7 & 0.08 & 7 & 0.09 & 0.05 \\
\hline \multirow[t]{2}{*}{ Secondary prophylaxis } & & & \multicolumn{2}{|c|}{ Cycle 2} & \multicolumn{2}{|c|}{ Cycle 3} & \multicolumn{2}{|c|}{ Cycle 4} & \multicolumn{2}{|c|}{ Cycle 5} & \multicolumn{2}{|c|}{ Cycle 6} & $\Delta P(\text { event })^{\mathrm{c}}$ \\
\hline & & & $n^{\mathrm{a}}$ & $P(\text { event })^{\mathrm{b}}$ & $n^{\mathrm{a}}$ & $P$ (event $^{\mathrm{b}}$ & $n^{\mathrm{a}}$ & $P(\text { event })^{\mathrm{b}}$ & $n^{\mathrm{a}}$ & $P(\text { event })^{\mathrm{b}}$ & $n^{\mathrm{a}}$ & 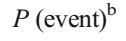 & \\
\hline \multicolumn{14}{|l|}{ Patients with } \\
\hline CIN4 & & & 18 & 0.08 & 6 & 0.11 & 2 & 0.12 & 2 & 0.14 & 2 & 0.16 & 0.07 \\
\hline $\mathrm{FN}$ & & & 9 & 0.04 & 2 & 0.05 & 0 & 0.05 & 1 & 0.06 & 0 & 0.06 & 0.02 \\
\hline Hospitalization & & & 10 & 0.05 & 1 & 0.05 & 1 & 0.06 & 1 & 0.06 & 0 & 0.06 & 0.02 \\
\hline Chemotherapy disturbance & & & & & 6 & 0.03 & 5 & 0.06 & 8 & 0.12 & 2 & 0.13 & 0.10 \\
\hline
\end{tabular}

${ }^{\mathrm{a}} n$ is the count of patients experiencing the event at each cycle

${ }^{\mathrm{b}} P$ (event) is the cumulative probability of having the event by a given cycle

c $\Delta P$ (event) is the change in probability of event from initiation cycle to cycle 6 (except for chemotherapy disturbance which has a one-cycle lag; see "Methods" section

resolved completely (95.9\%). The most frequent were bone pain $(23.0 \%)$, arthralgia $(14.2 \%)$, myalgia (7.4\%), diarrhea (6.8\%), back pain (4.7\%), and rash (4.7\%). Four serious ADRs (2.7\% of ADRs in 4 or $0.3 \%$ of patients) were reported, these being bone pain, drug hypersensitivity, vulval abscess, and loss of consciousness. There were no neutropenia-related and no Zarzio ${ }^{\circledR}$-related deaths.

\section{Discussion}

In this European study of patients with stage 3 or 4 cancer, treated with chemotherapy regimens with varying degrees of FN risk and receiving primary or secondary prophylaxis with Zarzio $^{\circledR}$, the rates of CIN4 and FN episodes, associated hospitalizations and chemotherapy disturbances, adverse events, and ADRs were statistically within the range of rates reported historically [16, 28, 29]. This documents the effectiveness and safety of Zarzio ${ }^{\circledR}$ in daily practice and extends the efficacy and safety data from its clinical development program [19-21]. These effectiveness and safety findings should address prescribers' concerns about biosimilars [30]. More importantly, this study revealed the impact of significant variability in prophylaxis patterns, often divergent from the EORTC guidelines for GCSFs. More than a decade of clinical experience with filgrastim has translated into practice patterns that differ from the normative filgrastim regimen in randomized controlled trials; attempt to balance chemotoxicity-associated $\mathrm{CIN} / \mathrm{FN}$ risk, risk factors, patient safety, and clinician experience; and aim to achieve therapeutic efficiency.
Zarzio ${ }^{\circledR}$ was used mainly for 5 days of primary prophylaxis at a dose of $30 \mathrm{MIU}$, which tended to be increased to $48 \mathrm{MIU}$ in function of weight, chemotoxicity, and tumor type. Almost six out of ten oncology patients were initiated in the EORTC-recommended time window of 24-72 $\mathrm{h}$ post-chemotherapy compared to one third of hematology patients. The latter tended to be started on prophylaxis between days 5 and 8 . Patients on a regimen with $<10 \%$ risk or on secondary prophylaxis tended to be prophylacted sooner. The modal and median durations of prophylaxis were 5 days, considerably shorter than the 10-11 days in trials, consistent with early findings from claim databases [31], and thus reflective of wellestablished CIN/FN prophylaxis patterns. Over a quarter of patients received secondary prophylaxis, which is essential in patients with a neutropenic event in a previous cycle. Yet, this relatively high proportion, coupled with the $17.4 \%$ under-prophylaxis data, suggests that, still, too many patients received secondary prophylaxis when primary prophylaxis was indicated. The low rate of antibiotic prophylaxis is likely due to the limited number of hematological malignancy patients, who have been shown to benefit from such prophylaxis [32].

Significant proportions of patients were under- $(17.4 \%)$ or over-prophylacted $(26.0 \%)$. Under-prophylaxis concerned patients on high FN risk chemotherapy or patients on moderate $\mathrm{FN}$ risk regimens but with $\mathrm{FN}$ risk factors. This is inconsistent with established evidence, further compounded by the increasingly shorter duration of prophylaxis. The overprophylaxis involved patients on low-chemotoxicity regimens or patients on moderate-risk regimens but without risk factors. This may signal a real-world trend for clinicians to "over- 
Table 5 Tolerability and safety

Patients $^{\mathrm{a}}$

$\%$ Patients

Events $^{\mathrm{b}}$

$\%$ Events

Clinical events (not mutually exclusive)

Musculoskeletal

Bone pain

Muscle pain

Joint pain

Hematological

Elevated laboratory values

Neurological

Splenomegaly

Death

Adverse drug reactions (ADR)

Of which:
Incidence of ADRs

Of which classified as serious

ADR intensity

Treatment

Cancer-related

Cancer-unrelated

Unknown

Mild

Moderate

Severe

Not reported

None

Medical

Non-medical

Not reported

Outcome
Resolved completely

Resolved with sequelae

On-going

Not reported
357

210

200

230

29

8

9

30

222

178

168

88

100

43

102

13

Patients

61

41

14

6

Patients $^{\mathrm{c}}$

76

4
$15.9 \%$

$2.0 \%$

$0.6 \%$

$0.6 \%$

$2.1 \%$

$15.3 \%$

$12.3 \%$

$11.6 \%$

$6.1 \%$

$6.9 \%$

$3.0 \%$

$7.1 \%$

$0.9 \%$

$\%$ Patients

$4.1 \%$

$67.2 \%$

$22.9 \%$

$9.9 \%$

$\%$ Patients

$5.1 \%$

$0.3 \%$
$7.2 \%$

$0.5 \%$

$0.2 \%$

$0.1 \%$

$0.5 \%$

$6.0 \%$

$4.8 \%$

$4.7 \%$

$2.0 \%$

$2.4 \%$

$0.8 \%$

$2.5 \%$

$0.3 \%$

$\begin{array}{lll}24.7 \% & 862 & 11.4 \% \\ 14.5 \% & 519 & 6.9 \% \\ 13.8 \% & 453 & 6.0 \%\end{array}$

$\begin{array}{ll}\text { Events }^{\mathrm{d}} & \% \text { Events } \\ 148 & 2.3 \% \\ 4 & <0.1 \% \\ \text { Events }^{\mathrm{e}} & \% \text { Events }^{\mathrm{e}}\end{array}$

66

58

8

16

89

50

6

3

142

1

1

4

$44.6 \%$

$39.2 \%$

$5.4 \%$

$10.8 \%$

$60.1 \%$
$33.8 \%$
$4.1 \%$
$2.0 \%$

$42 \quad 95.9 \%$

$0.7 \%$

$0.7 \%$

$2.7 \%$ 
Table 5 (continued)

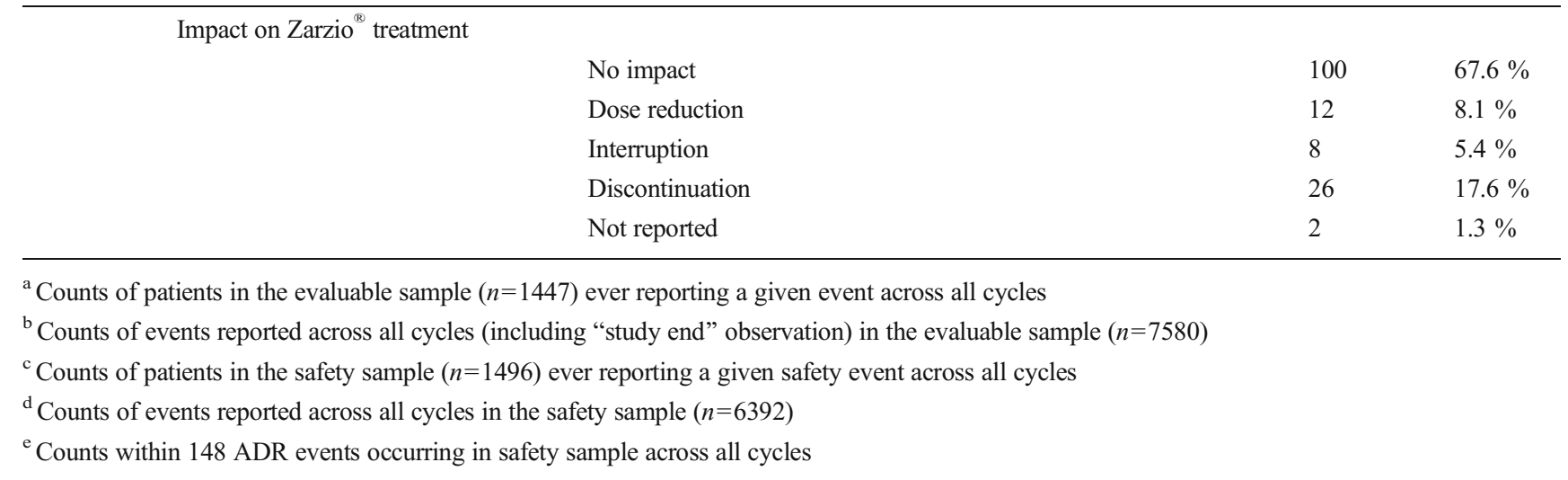

protect" and reflect a change in practice that heretofore has not been described in real-world studies. If confirmed in future studies, the over-prophylaxis pattern may result in modifications of existing prophylaxis paradigms. The underprophylaxis patterns are a reminder that, despite risk stratification and evidence-based guidelines, a significant proportion of patients may receive inadequate CIN/FIN prophylaxis.

One in seven patients received their first dose of Zarzio ${ }^{\circledR}$ on the day of chemotherapy. While the evidence is limited, derived mainly from non-controlled studies of pegfilgrastim, and often contradictory in findings, NCCN guidelines now cautiously acknowledge that "same-day administration may be considered in certain circumstances" [15]. The cyclelevel analyses showed that same-day initiation was not associated with higher $\mathrm{CIN}$ rates.

In both the patient-level and cycle-level analyses, type and duration of prophylaxis, day of initiation, and FN risk were found to impact the CIN $1 / 4, \mathrm{CIN} 3 / 4$, CIN4, and FN rates; CIN/FN-related hospitalizations and chemotherapy disruptions; and the composite outcome. This is consistent with prior evidence $[4,5,16,24]$.

Seemingly paradoxical, higher rates of CIN, FN, and $\mathrm{CIN} / \mathrm{FN}$-related outcomes were found in cycles in which Zarzio $^{\circledR}$ was administered for $\geq 6$ days. However, duration was longer in patients on regimens with $\mathrm{FN}$ risk $>20 \%$ and presenting with additional risk factors. This may reflect clinician vigilance about chemotoxicity and risk factors and a goal of optimizing outcomes. Noteworthy, rates for chemotherapy disturbances, in general, and for chemotherapy delays specifically were at least twice as high in patients on secondary prophylaxis, thus exposing these patients to the risk of impaired tumor control.

The rates of musculoskeletal, hematological, elevated laboratory values, neurological, and splenomegaly events were consistent with the known safety profile for GCSFs. Likewise, ADR rates were consistent with known rates.
The use of biosimilar filgrastim offers significant cost savings over standard filgrastim (Neupogen ${ }^{\circledR}$ ) or pegfilgrastim (Neulasta ${ }^{\circledR}$ ). In fact, an economic analysis for the European Union (EU) G5 countries comparing 1 to 14 days of prophylaxis with Zarzio ${ }^{\circledR}$ versus 1 to 14 days with Neupogen ${ }^{\circledR}$ or a singular injection with Neulasta ${ }^{\circledR}$ showed, that under all scenarios, Zarzio ${ }^{\circledR}$ was the most cost-efficient approach to prophylaxis [33]. Further, savings achieved with conversion to biosimilars can be applied, in a budget-neutral way, to purchase additional curative anticancer therapy, and this expands patient access to such treatments as rituximab for diffuse large B cell non-Hodgkin's lymphoma and trastuzumab for metastatic HER2-positive breast cancer. For the EU G5 countries, it has been estimated that, depending on regimen, such expanded access to rituximab can be achieved by converting between 4 and 14 patients from Neupogen ${ }^{\circledR}$ and between 2 and 24 patients from Neulasta ${ }^{\circledR}$ to Zarzio ${ }^{\circledR}$. Likewise, expanded access to trastuzumab would require converting between 4 and 14 patients from Neupogen ${ }^{\circledR}$ and between 2 and 24 patients from Neulasta ${ }^{\circledR}[34]$.

Our study has limitations. It was not a population-based study, was limited to centers using Zarzio ${ }^{\circledR}$, and included only patients prophylacted with this biosimilar. The investigators selected patients, and this may be a source of selection bias. Despite strict inclusion and exclusion criteria, most patients had no or minimal impairment in performance status, and about a quarter had experienced a CIN or FN episode in a prior chemotherapy line or cycle. It is possible that investigators showed preference for less-impaired patients with better prognosis or patients with a prior CIN/FN history and therefore candidates for prophylaxis - some of whom may have been over-prophylacted relative to the EORTC guidelines. The design did not permit calculation of the relative dose intensity (RDI) of chemotherapy regimens, which has been shown to be associated with all-cause mortality [4]. Further analysis is needed to identify determinants of the CIN/FN 
incidence, hospitalization, and chemotherapy disturbance rates and to compare specific subgroups such as oncological vs. hematological patients; elderly vs. non-elderly patients; under-, correctly, and over-prophylacted patients; and patients with same-day vs. $24-72$-h vs. $>72$-h initiation of prophylaxis.

\section{Conclusion}

The clinical and safety outcomes of prophylaxis with biosimilar filgrastim are within the range of historically reported data for originator filgrastim. This underscores not only the clinical effectiveness and safety of biosimilar filgrastim prophylaxis in daily clinical practice, but also the need to improve primary prophylaxis rates in patients with chemotherapyrelated and patient-related risk factors for FN.

Acknowledgments The MONITOR-GCSF study was funded by Sandoz Biopharmaceuticals. Sponsor participated in the development of the protocol, the discussion of the results, and review of the manuscript for scientific content.

Conflict of interest P.G., M.A., H.L., C.B., and M.B. received compensation from Sandoz Biopharmaceuticals for their participation in the work reported here. M.T. is an employee of Sandoz Biopharmaceuticals. K.D., K.M., and I.A. are affiliated with Matrix45. By company policy, they cannot hold equity in sponsor organizations and cannot receive direct personal benefits, financial or other, from sponsor organizations. Matrix 45 provides similar services to other biopharmaceutical companies without exclusivity constraints.

\section{References}

1. Lalami Y, Paesmans M, Muanza F et al (2006) Can we predict the duration of chemotherapy-induced neutropenia in febrile neutropenic patients, focusing on regimen-specific risk factors? A retrospective analysis. Ann Oncol 17:507-514

2. Lalami Y, Paesmans M, Aoun M et al (2004) A prospective randomized evaluation of G-CSF or G-CSF plus oral antibiotics in chemotherapy-treated patients at high risk of developing febrile neutropenia. Support Care Cancer 12:725-730

3. Lyman GH, Lyman CH, Agboola O (2005) Risk models for predicting chemotherapy-induced neutropenia. Oncologist 10: 427-437

4. Lyman GH, Dale DC, Culakova E et al (2013) The impact of the granulocyte colony-stimulating factor on chemotherapy dose intensity and cancer survival: a systematic review and meta-analysis of randomized controlled trials. Ann Oncol 24:2475-2484

5. Lyman GH, Abella E, Pettengell R (2014) Risk factors for febrile neutropenia among patients with cancer receiving chemotherapy: a systematic review. Crit Rev Oncol Hematol 90:190-199

6. Kuderer N, Dale D, Crawford J et al (2007) Impact of primary prophylaxis with granulocyte colony-stimulating factor or febrile neutropenia and mortality in adult cancer patients receiving chemotherapy: a systematic review. J Clin Oncol 25:3158-3167

7. Crawford J, Dale D, Lyman GH (2003) Chemotherapy-induced neutropenia. Cancer 100:228-237
8. Komorokji R, Lyman G (2004) The colony-stimulating factors: use to prevent and treat neutropenia and its complications. Exp Opin Biol Ther 4:1897-1910

9. Aapro M, Cameron D, Pettengell R et al (2006) EORTC guidelines for the use of granulocyte-colony stimulating factor to reduce the incidence of chemotherapyinduced febrile neutropenia in adult patients with lymphomas and solid tumours. Eur J Cancer 42:24332453

10. Aapro MS, Bohlius J, Cameron DA et al (2011) 2010 update of EORTC guidelines for the use of granulocyte-colony stimulating factor to reduce the incidence of chemotherapy-induced febrile neutropenia in adult patients with lymphomas and solid tumours. Eur J Cancer 47:8-32

11. Gridelli C, Aapro M, Barni S et al (2007) Role of colony stimulating factors (CSFs) in solid tumours: results of an expert panel. Crit Rev Oncol Hematol 63:53-64

12. Raposo CG, Marin AP, Barón MG (2006) Colony-stimulating factors: clinical evidence for treatment and prophylaxis of chemotherapy-induced febrile neutropenia. Clin Transl Oncol 8:729-734

13. Wingard JR, Elmongy M (2009) Strategies for minimizing complications of neutropenia: Prophylactic myeloid growth factors or antibiotics. Crit Rev Oncol Hematol 72:144-154

14. Morrison V, Wong M, Hershman D et al (2007) Observational study of the prevalence of febrile neutropenia in patients who received filgrastim or pegfilgrastim associated with 3-4 week chemotherapy regimens in community oncology practices. J Manag Care Pharm 13:337-348

15. 15 National Comprehensive Center Network. NCCN Clinical Practice Guidelines in Oncology (NCCN Guidelines $\left.{ }^{\circledR}\right)$. Myeloid growth factors version 2.2014. Available at http://www.nccn.org/ professionals/physician_gls/pdf/myeloid_growth.pdf. Accessed 2 Oct 2014

16. Klastersky J, Awada A (2011) Prevention of febrile neutropenia in chemotherapytreated cancer patients: Pegylated versus standard myeloid colony stimulating factors. Do we have a choice? Crit Rev Oncol Hematol 78:17-23

17. Klastersky J, Awada A, Paesmans M, Aoun M (2011) Febrile neutropenia: a critical review of the initial management. Crit Rev Oncol Hematol 78:185-194

18. Weise M, Bielsky MC, De Smet K et al (2011) Biosimilars - why terminology matters. Nat Biotechnol 29:690-693

19. Gascón P, Fuhr U, Sörgel F et al (2010) Development of a new GCSF product based on biosimilarity assessment. Ann Oncol 21: 1419-1429

20. Abraham I, Tharmarajah S, MacDonald K (2013) Clinical safety of biosimilar recombinant human granulocyte colony stimulating factors. Exp Opin Drug Saf 12:235-246

21. Tharmarajah S, Mohammed A, Bagalagel A et al (2014) Clinical efficacy and safety of Zarzio (EP2006), a biosimilar recombinant human granulocyte colony stimulating factor. Biogeosciences $4: 1-$ 9

22. Verpoort K, Möhler TM (2012) A non-interventional study of biosimilar granulocyte colony-stimulating factor as prophylaxis for chemotherapy-induced neutropenia in a community oncology centre. Ther Adv Med Oncol 4:289-293

23. Salesi N, Di Cocco B, Veltri E (2012) Biosimilar medicines in oncology: singlecenter experience with biosimilar G-CSF. Fut Oncol 8:625-630

24. Gascón P, Tesch H, Verpoort K et al (2013) Clinical experience with Zarzio $^{\circledR}$ in Europe: what have we learned? Support Care Cancer 21: 2925-2932

25. Bonig H, Becker PS, Schwebig A, Turner M (2015) Biosimilar granulocyte-colonystimulating factor for healthy donor stem cell mobilization: need we be afraid? Transfusion 55:430-439 
26. Gascón P, Aapro M, Ludwig H et al (2011) Background and methodology of MONITOR-GCSF, a pharmaco-epidemiological study of the multi-level determinants, predictors, and clinical outcomes of febrile neutropenia prophylaxis with biosimilar granulocyte-colony stimulating factor filgrastim. Crit Rev Oncol Hematol 77:184-197

27. Gascón P, Aapro M, Ludwig H et al (2011) Update on the MONITOR-GCSF study of biosimilar filgrastim to reduce the incidence of chemotherapy-induced febrile neutropenia in cancer patients: Protocol amendments. Crit Rev Oncol Hematol 77:198-200

28. Kuderer N, Dale D, Crawford J et al (2007) Impact of primary prophylaxis with granulocyte colony-stimulating factor on febrile neutropenia and mortality in adult cancer patients receiving chemotherapy: a systematic review. J Clin Oncol 25:3158-67

29. 29 European Medicines Agency. Zarzio. Annex I. Summary of product characteristics. Available at http://www.ema.europa.eu/ docs/en_GB/document_library/EPAR__Product_Information/ human/000917/WC500046525.pdf. Accessed 22 Nov 2014
30. Aapro MS (2012) What do prescribes think of biosimilars? Targ Oncol 7(Suppl 1):S51-S55

31. Weycker D, Hackett J, Edelsberg JS et al (2006) Are shorter courses of filgrastim prophylaxis associated with increased risk of hospitalization? Ann Pharmacother 40:402-407

32. Gafter-Gvili A, Fraser A, Paul M et al (2012) Antibiotic prophylaxis for bacterial infections in afebrile neutropenic patients following chemotherapy. Cochrane Database Syst Rev 201, CD004386

33. Aapro M, Cornes P, Abraham I (2011) Comparative cost-efficiency across the European G5 countries of various regimens of filgrastim, biosimilar filgrastim, and pegfilgrastim to reduce the incidence of chemotherapy-induced febrile neutropenia. J Oncol Pharm Pract 18:171-179

34. Sun D, Andayani TM, Altyar A et al (2015) Potential cost savings from chemotherapyinduced febrile neutropenia prophylaxis with biosimilar filgrastim and expanded access to targeted antineoplastic treatment across the European G5 countries: a simulation study. Clin Ther 37:842-857 